Open Access

\title{
Molecular essence and endocrine responsiveness of estrogen receptor-negative, progesterone receptor-positive, and HER2- negative breast cancer
}

\author{
Ke-Da Yu*, Yi-Zhou Jiang, Shuang Hao and Zhi-Ming Shao*
}

\begin{abstract}
Background: The clinical significance of progesterone receptor (PgR) expression in estrogen receptor-negative (ER-) breast cancer is controversial. Herein, we systemically investigate the clinicopathologic features, molecular essence, and endocrine responsiveness of ER-/PgR+/HER2- phenotype.
\end{abstract}

Methods: Four study cohorts were included. The first and second cohorts were from the Surveillance, Epidemiology, and End Results database $(n=67,932)$ and Fudan University Shanghai Cancer Center $(n=2,338)$, respectively, for clinicopathologic and survival analysis. The third and fourth cohorts were from two independent publicly available microarray datasets including 837 operable cases and 483 cases undergoing neoadjuvant chemotherapy, respectively, for clinicopathologic and gene-expression analysis. Characterized genes defining subgroups within the ER-/PgR+/HER2- phenotype were determined and further validated.

Results: Clinicopathologic features and survival outcomes of the ER-/PgR+ phenotype fell in between the $\mathrm{ER}+/ \mathrm{PgR}+$ and ER-/PgR- phenotypes, but were more similar to ER-/PgR-. Among the ER-/PgR+ phenotype, $30 \%$ (95\% confidence interval [CI] 17-42\%, pooled by a fixed-effects method) were luminal-like and $59 \%$ (95\% Cl 45-72\%, pooled by a fixed-effects method) were basal-like. We further refined the characterized genes for subtypes within the ER-/PgR+ phenotype and developed an immunohistochemistry-based method that could determine the molecular essence of ER-/PgR+ using three markers, TFF1, CK5, and EGFR. Either PAM50-defined or immunohistochemistry-defined basal-like ER-/PgR+ cases have a lower endocrine therapy sensitivity score compared with luminal-like ER-/PgR+ cases $(P<0.0001$ by Mann-Whitney test for each study set and $P<0.0001$ for pooled standardized mean difference in meta-analysis). Immunohistochemistry-defined basal-like ER-/PgR+ cases might not benefit from adjuvant endocrine therapy (log-rank $P=0.61$ for sufficient versus insufficient endocrine therapy).

Conclusions: The majority of ER-/PgR+/HER2- phenotype breast cancers are basal-like and associated with a lower endocrine therapy sensitivity score. Additional studies are needed to validate these findings.

Keywords: Basal-like, Breast cancer, ER-/PgR+, Endocrine responsiveness, Molecular subtype

\footnotetext{
*Correspondence: yukeda@163.com; zhimingshao@yahoo.com

Department of Breast Surgery, Cancer Center and Cancer Institute,

Shanghai Medical College, Fudan University, 399 Ling-Ling Road, Shanghai

200032, P. R. China
} 


\section{Background}

The progesterone receptor (PgR) is a downstream relative of the estrogen receptor (ER), which activates the expression of PgR via the estrogen-responsive element located in the promoter region of the PgR gene. Adequate expression of PgR indicates a functional ER- $\alpha$ and ER- $\alpha$ pathway [1]. Loss of PgR expression in ER-positive $(\mathrm{ER}+)$ breast cancer potentially defines a subgroup with impaired function in the ER pathway, which probably gains limited benefit from endocrine therapy [2-4].

Clinically, it is generally agreed upon that all newlydiagnosed primary breast cancers should be evaluated for ER and PgR protein expression by immunohistochemistry (IHC). Although some researchers have suggested that the ER-negative/PgR-positive (ER-/PgR+) phenotype does not actually exist and may represent technical artifacts [5-7], an increasing body of evidence has shown that ER-/PgR+ tumors exist both biologically and clinically [8, 9]. Moreover, an ER-/PgR+ breast cancer cell line had been described earlier [10], indicating a mechanism of PgR expression regulation independent from ER- $\alpha$.

The recently updated St. Gallen consensus on early-stage breast cancer recommends making clinical treatment decisions based on the surrogates of molecular subtypes (luminal-A, luminal-B, HER2-positive, and basal-like) defined by ER, PgR, HER2, and Ki67 [11]. The St. Gallen panelists failed to categorize the ER-/PgR+/HER2phenotype into the four molecular subtypes, while some other guidelines treated the ER-/PgR+/HER2- phenotype as a luminal-B subtype. The ER-/PgR+ group accounts for $1-5 \%$ of all breast cancers $[2,8]$. Even after repeated reassessment of ER and PgR in these cases as the American Society of Clinical Oncology/College of American Pathologists (ASCO/CAP) guidelines recommend [12], at least $50 \%$ of ER-/PgR+ remained $[2,5]$.

Some efforts have been made to reveal the molecular essence of ER-/PgR+ breast cancer. Using gene-expression profile information, Itoh et al. [13] proposed that, among these patients, $20 \%$ were luminal-like and $65 \%$ were basallike, indicating for the first time that $\mathrm{ER}-/ \mathrm{PgR}+$ breast cancer is a mixed group. In the current study, we included four large cohorts of breast cancer cases and systemically studied the clinical features and molecular essence of the ER-/PgR+ phenotype. Furthermore, we established a feasible and reliable IHC-based method to determine the subtype of each ER-/PgR+ case to guide individualized treatment. Because HER2+ breast cancers represent a biologically distinct subgroup [14], we excluded HER2 + cases from this study.

\section{Methods}

\section{Four study cohorts}

Cohort 1 was obtained from the database of the Surveillance, Epidemiology, and End Results (SEER) program in the United States. Cohort 2 was retrieved from the Fudan University Shanghai Cancer Center (FDUSCC). Cohort 3 was a publicly available gene expression microarray dataset previously published elsewhere [15]. Cohort 4 was also a publicly available dataset including patients undergoing neoadjuvant chemotherapy [16]. The basic characteristics of the four cohorts are shown in Table 1. The study flowchart diagram is shown in Additional file 1: Figure S1. In addition, we analyzed 64 consecutive cases with the ER-/PgR+/HER2- phenotype from FDUSCC between 2005 and 2011 to validate IHC-based markers of subtype classification (characteristics of the 64 cases are available in Additional file 2: Table S1). The datasets (cohorts 1, 3, and 4) we used in this study are publically available and no permissions were required. The research protocols of cohorts 1,3 , and 4 were determined to be qualified for institutional review board exemption by the Ethical Committee of the Shanghai Cancer Center of Fudan University. The research protocols for cohort 2 and 64 consecutive ER-/PgR+/HER2- cases were reviewed and approved by the Ethical Committee of the Shanghai Cancer Center of Fudan University. All participants provided written informed consents.

For cohort 1, obtained from the SEER database consisting of 18 population-based cancer registries, we selected patients diagnosed with invasive breast cancer between January 1, 2010, and December 31, 2013 (SEER provides HER2 status after 2010). We identified 67,932 HER2negative patients according to the following criteria: female, surgical treatment (either mastectomy or breastconservation), AJCC stages I-III, pathologically confirmed invasive ductal carcinoma, unilateral, known ER/PgR/ HER2 status, known time of diagnosis, and breast cancer as the first cancer at diagnosis. SEER database does not conduct central review for ER/PgR/HER2. Since we enrolled the cases after 2010, the positivity of ER and PR expression should be according to the ASCO/CAP guideline ( $\geq 1 \%$ of tumor cells with nuclear staining) [12]. Data extraction was performed by SEER*Stat software v8.1.5 [17]. The outcome of interest was breast cancer-specific survival (BCSS), which was calculated from the date of diagnosis to the date of breast cancer death. Patients who died of other causes were censored at the date of death.

For cohort 2 from FDUSCC, we included 2,338 consecutive HER2- cases of primary operable invasive breast cancer between January 1, 2008, and December 31, 2011. This is a well-characterized series of patients, whose clinicopathologic and follow-up information were maintained on a prospective basis [18]. Patient treatments were based on St. Gallen consensus $[11,19]$. The cut-off for ER/PgR positivity was $\geq 1 \%$ of tumor cells with nuclear staining [12]. Pathologic HER2 status was defined according to ASCO/CAP guidelines [20]. Re-assessment of ER-/PgR+ 
Table 1 Clinicopathologic characteristics of patients with HER2-negative breast cancer included for analysis

\begin{tabular}{|c|c|c|c|c|c|c|c|c|}
\hline \multirow[t]{2}{*}{ Characteristics } & \multicolumn{2}{|c|}{ Cohort 1: SEER } & \multicolumn{2}{|c|}{ Cohort 2: FDUSCC } & \multicolumn{2}{|c|}{ Cohort 3: Publicly available cases } & \multicolumn{2}{|c|}{ Cohort 4: Publicly available NCT cases } \\
\hline & $N=67,932$ & $\%$ & $\mathrm{~N}=2,338$ & $\%$ & $\mathrm{~N}=837$ & $\%$ & $N=483$ & $\%$ \\
\hline Age, years (IQR) & $61(51-70)$ & & $53(45-60)$ & & $55(45-65)$ & & $50(42-58)$ & \\
\hline \multicolumn{9}{|l|}{ Tumor size } \\
\hline T0-1 & 42,281 & 62.2 & 1,132 & 48.4 & 155 & 38.8 & 33 & 6.8 \\
\hline $\mathrm{T} 2$ & 20,253 & 29.8 & 1,004 & 42.9 & 210 & 52.6 & 244 & 50.5 \\
\hline T3-4 & 5,398 & 7.9 & 202 & 8.6 & 34 & 8.5 & 206 & 42.7 \\
\hline \multicolumn{9}{|l|}{ Lymph nodes } \\
\hline Negative & 47,078 & 69.3 & 1,168 & 50.2 & 285 & 47.3 & 150 & 31.1 \\
\hline Positive & 20,854 & 30.7 & 1,161 & 49.8 & 317 & 52.7 & 333 & 68.9 \\
\hline \multicolumn{9}{|l|}{ Grade } \\
\hline I & 17,172 & 26.2 & 39 & 1.7 & 102 & 13.2 & 31 & 6.8 \\
\hline$\|$ & 29,359 & 44.7 & 1,610 & 68.9 & 244 & 31.6 & 173 & 38.1 \\
\hline III and UD & 19,129 & 29.1 & 689 & 29.5 & 426 & 55.2 & 250 & 55.1 \\
\hline \multicolumn{9}{|l|}{ Subgroup } \\
\hline $\mathrm{ER}+/ \mathrm{PgR}+$ & 50,679 & 74.6 & 1,686 & 72.1 & 391 & 46.7 & 216 & 44.7 \\
\hline $\mathrm{ER}+/ \mathrm{PgR}-$ & 7,075 & 10.4 & 177 & 7.6 & 130 & 15.5 & 72 & 14.9 \\
\hline ER-/PgR+ & 561 & 0.8 & 34 & 1.5 & 36 & 4.3 & 17 & 3.5 \\
\hline ER-/PgR- & 9,617 & 14.2 & 441 & 18.9 & 280 & 33.5 & 178 & 36.9 \\
\hline Median follow-up, months (IQR) & $11(5-17)$ & & $37(25-50)$ & & $49(20-72)$ & & $36(21-49)$ & \\
\hline
\end{tabular}

FDUSCC Fudan University Shanghai Cancer Center, IQR Interquartile range, NCT Neoadjuvant chemotherapy, SEER Surveillance, Epidemiology and End Results program, UD Undifferentiated

cases was carried out routinely. The outcome for this cohort was relapse-free survival (RFS), which was calculated from the date of diagnosis to the date of the first event of local, regional, or distant metastasis of breast cancer.

For cohort 3, retrieved from 36 publicly available breast cancer microarray datasets [15], among the original 5,715 unique breast cancer with expression profiles, 837 cases were identified to be HER2-negative and had information on immunohistochemical ER, PgR, and HER2 status. The normalization of gene expression data was performed by Haibe-Kains et al. [15]. Hybridization probes were mapped to Entrez GeneID as described by Shi et al. [21]. When multiple probes mapped to the same GeneID, the one with the highest variance was used. All untreated patients had surgery, although information was not available for all datasets. The PAM50 classifier was applied to the data to determine the intrinsic subtype of each case as previously described [22]. The survival outcome of interest was RFS.

For cohort 4, we selected 483 HER2- patients who participated in a prospective Institutional Review Boardapproved biomarker discovery study at MD Anderson Cancer Center as published previously elsewhere [16]. The cut-off for ER/PgR positivity was $\geq 1 \%$ of tumor cells with nuclear staining. All patients received neoadjuvant chemotherapy containing a taxane/anthracycline-based regimen (followed by endocrine therapy if ER+). In our analysis, cases with indeterminate ER and PgR had been excluded, and the outcome for analysis was distant RFS (DRFS). Detailed methods for RNA purification and microarray hybridization have been reported previously $[16,23]$. Gene expression profiling with Affymetrix U133 gene chips was performed. Gene expression levels were derived from multiple oligonucleotide probes on the microarray that hybridize to different sequence sites of a gene transcript (probe sets). Gene expression data are available under Gene Expression Omnibus accession number of GSE25066. The PAM50 classifier was applied to determine the subtype of each case [22].

\section{Gene expression measurement}

In cohorts 3 and 4, each ER-/PgR+ case was assigned an intrinsic subtype by the PAM50 classifier [22]. The original gene expression profile data were only available in cohort 4 . Using these original data [16], we compared the gene expressions of interest between different subtypes of ER-/PgR+. To determine the functional ER pathway, mRNA expression of estrogen-responsive genes, TFF1 (pS2), GREB1, and PDZK1, were measured [24]. Expression of basal-associated cytokeratins (CKs) and EGFR were measured $[25,26]$. Moreover, because the claudin-low subgroup is associated with a specific subtype of triple-negative breast cancer, mesenchymal stem-like [27], we also measured the expression of the epithelial-mesenchymal transitionassociated gene $\mathrm{CDH} 1$ and claudin genes to discriminate 
mesenchymal stem-like from basal-like [28]. Probe sets used for measurement of mRNA expression are listed in Additional file 3: Table S2. Expression data were normalized with the MAS5 algorithm, the mean was centered to 600 and $\log 2$ was transformed as previously described [13].

An endocrine therapy sensitivity score was calculated by the average $\log 2$ transformed expression values of ER, PgR, BCL2, and SCUBE2 with following measurement: $\left(0.8 * \mathrm{ER}+1.2^{*} \mathrm{PGR}+\mathrm{BCL} 2+\mathrm{SCUBE} 2\right) / 4$ as previously described in OncotypeDX [29]. This ER group score could predict of response to tamoxifen and a higher score indicates a higher sensitivity to endocrine therapy [30, 31]. For 64 cases (consecutive cases with the ER-/PgR+/HER2- phenotype from FDUSCC) with formalin-fixed paraffin-embedded samples, the method of RNA extraction and real-time PCR is provided in Additional file 4: Supplemental Methods. PCR primers are listed in Additional file 5: Table S3.

\section{Immunohistochemistry}

IHC was performed in the 64 cases from FDUSCC according to the standard procedure [25]. Staining patterns were as follows: cytoplasmic and/or membranous staining for EGFR and CK5, and cytoplasmic staining for TFF1 (pS2). The cutoff value for positivity for TFF1 was $10 \%$ [32]; CK5 and EGFR scored positive if any (weak or strong) staining was observed as previously described [25]. The antibodies used were reported in our previous study [33].

\section{Statistical analysis}

Comparisons of patient and tumor characteristics were performed using the $\chi^{2}$ test or two-sample $t$-test. Survival curves were constructed using Kaplan-Meier method and tested by log-rank test. Multivariate adjusted hazard ratios (HRs) with $95 \%$ confidence intervals (CIs) were calculated using the Cox proportional hazards model. The MannWhitney test was used to test gene expression differences. To analyze the combined results, we employed a two-step approach [34]. At first, the individual participant data from each study were analyzed separately (i.e. to obtain the results of each cohort). Then, the results were synthesized in the second step using a suitable model for meta-analysis of aggregate data. The meta-analysis was conducted in adherence to the standards of quality [35]. To pool the proportions, we used the command "metaprop_one" in Stata. According to a previous study [36], the score methods are recommended for proportion interval estimates and in our study the Wilson score confidence intervals were computed. We also assessed the heterogeneity among cohorts by using Cochran $X^{2}$ $\mathrm{Q}$ statistics and $I^{2}$ statistics. If $P$ values $<0.05$ or $I^{2}>25 \%$ were obtained, we determined that there was a significant heterogeneity [35]. Use of a fixed-effects method (Inversevariance method) or a random-effects method (DerSimonian and Laird method) was performed according to heterogeneity. When we compared survival estimates of ER-/PgR+ versus ER+/PgR+ and ER-/PgR- versus $\mathrm{ER}+/ \mathrm{PgR}+$, we used multivariate meta-analysis (command "mvmeta" in Stata). Multivariate meta-analysis has been described previously $[37,38]$. The method we used was restricted maximum likelihood and the variance-covariance matrix was defined as "unstructured". Statistical analyses were performed with Stata v.14.0 and SPSS v.17. Twosided $P<0.05$ was considered statistically significant.

\section{Results}

Clinicopathologic features and survival outcomes of breast cancer with ER-/PgR+/HER2- phenotype

In HER2- cases, the ER-/PgR+ phenotype accounted for $0.8-4.3 \%$ among the four cohorts, with the pooled overall proportion of $2.5 \%$ (95\% CI, 1.4-3.6 \%, by a random-effects method), which is consistent with previous reports $[2,8,9,39]$. Of note, in the consecutive cases from cohorts 1 and 2, the overall proportion of the ER-/PgR+ phenotype was $1.1 \%$ (95\% CI, 0.5-1.7\%, by a random-effects method).

We compared the clinicopathologic characteristics of tumors of the ER-/PgR+ phenotype with those of the $\mathrm{ER}+/ \mathrm{PgR}+$ and ER-/PgR- phenotypes (Additional file 6: Table S4). ER-/PgR+ tumors were associated with significantly younger age at onset, larger tumor size, higher positive node rate, and higher grade (all $P<0.001$ ) compared with $\mathrm{ER}+/ \mathrm{PgR}+$ tumors in cohort 1 . These differences were successfully validated in most but not all the other cohorts. For instance, difference in tumor size observed in cohort 1 failed to be validated in cohort 3 . When compared with the ER-/PgR- phenotype, ER-/PgR+ tumors showed characteristics that were similar to or slightly more favorable than those of the ER-/PgR- phenotype.

Significant differences in survival between ER-/PgR+ and $\mathrm{ER}+/ \mathrm{PgR}+$ were observed in cohorts $1-3$ but not in cohort 4 either in univariate analysis (Fig. 1) or after adjustment (cohort 1: HR $=3.26$ [95 \% CI, 1.71-6.22], $P<0.001$ for BCSS after adjustment for age, tumor size, lymph nodes status, and grade; cohort 2: $\mathrm{HR}=2.61$ [95 \% CI, 1.20-5.67], $P=0.016$ for RFS after adjustment for age, tumor size, lymph nodes status, and grade; cohort 3: HR $=2.68$ [95 \% CI, 1.10-6.55], $P=0.030$ for RFS after adjustment for age, lymph nodes status, and grade; cohort 4: HR $=1.09$ [95\% CI, 0.26-4.64], $P=0.90$ for DRFS after adjustment for age, tumor size, lymph nodes status, grade, and pathological complete response; Table 2). In contrast, there were numerical but insignificant differences between $\mathrm{ER}-/ \mathrm{PgR}+$ and ER-/PgR - phenotypes. Generally, ER-/PgR+ showed survival outcomes midway between $\mathrm{ER}+/ \mathrm{PgR}+$ and $\mathrm{ER}-/ \mathrm{PgR}-$, although the survival curve of $\mathrm{ER}-/ \mathrm{PgR}+$ was more similar 

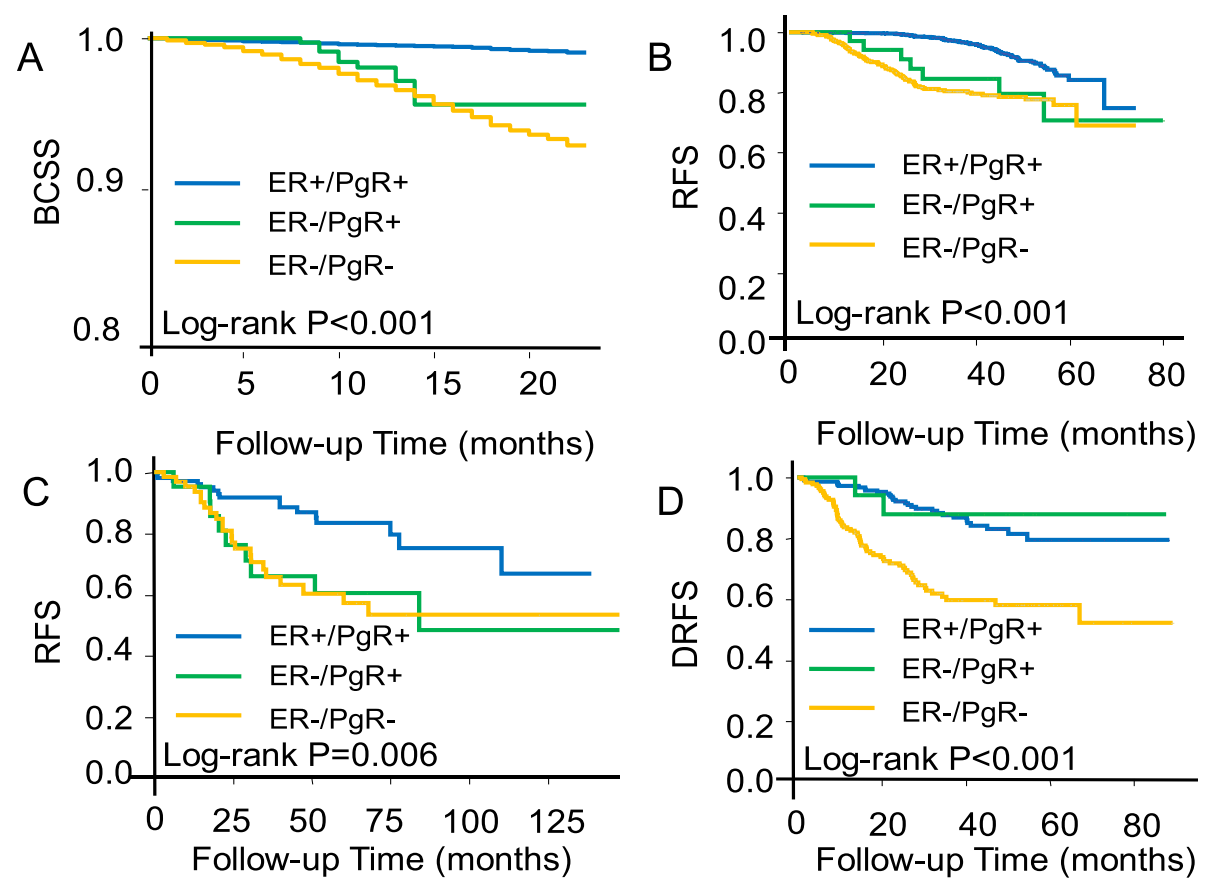

Fig. 1 Kaplan-Meier estimates of survival are shown according to ER and PgR status in the four cohorts. (a) Breast cancer-specific survival (BCSS) of cohort 1; (b) Relapse-free survival (RFS) of cohort 2; (c) RFS of cohort 3; (d) Distant relapse-free survival (DRFS) of cohort 4. Log-rank $P$ values are shown

to that of the ER-/PgR- cases. The fact that survival outcomes in cohorts $1-3$ could not be observed in cohort 4 might be because of limited number of ER-/PgR+ cases $(\mathrm{n}=17)$ and highly selected patients (with locally advanced disease and who underwent neoadjuvant chemotherapy) in that cohort. Furthermore, we investigated the pooled survival outcomes of $\mathrm{ER}-/ \mathrm{PgR}+$ versus $\mathrm{ER}+/ \mathrm{PgR}+$ and $\mathrm{ER}-/ \mathrm{PgR}-$ versus $\mathrm{ER}+/ \mathrm{PgR}+$ by using multivariate metaanalysis (method: restricted maximum likelihood), the pooled HR was 2.67 (95\% CI, 1.77-4.05) for ER-/PgR+ versus $\mathrm{ER}+/ \mathrm{PgR}+$ and 3.97 (95\% CI, 3.38-4.66) for $\mathrm{ER}-/ \mathrm{PgR}-$ versus $\mathrm{ER}+/ \mathrm{PgR}+$. Taken together, the clinicopathologic features and survival outcomes of the ER-/PgR+ phenotype fell in between the ER+/PgR+ and ER-/ $\mathrm{PgR}$ - groups but were closer to the latter.

\section{Intrinsic molecular subtypes within ER-/PgR+/HER2- phenotype}

Intrinsic molecular subtypes of breast cancer have been thoroughly studied $[14,40]$, but previous research failed to assign the ER-/PgR+ phenotype to one specific and stable molecular subtype [41]. Cohorts 3 and 4, but not cohorts 1 and 2, had available information of intrinsic molecular subtypes defined by gene expression profile. We therefore explored the distribution of known intrinsic subtypes within the ER-/PgR+ phenotype in cohorts 3 and 4 . Independent cohorts 3 and 4 showed similar results and the ER-/PgR+ phenotype had a higher likelihood of being the basal-like subtype (Table 3). When we combined these two cohorts together using a meta-analytic approach (command "metaprop_one" in Stata), 30 \% (95 \% CI, 17$42 \%$, by a fixed-effects method) of ER-/PgR+ phenotype was luminal-like and $59 \%$ (95\% CI, 45-72\%, by a fixedeffects method) were basal-like. Both the luminal-like and basal-like subtypes accounted for about $89 \%$ of the whole $\mathrm{ER}-/ \mathrm{PgR}+$ group.

Because we had the original gene expression data of each case in cohort 4, we could investigate the ESR1 gene (ER) expression in the ER-/PgR+ phenotype in this cohort. A $\log 2$-transformed expression value of $\geq 10.18$ was considered as ER+ by mRNA according to a threshold established in previous publications [13, 23]. Five of 17 ( $29 \%$; $95 \%$ CI, 10-56 \%) patients who were IHC ER- had high expression of ESR1 mRNA and may be considered as false-negative IHC results. The majority of the ER-/PgR+ phenotype $(71 \%)$ showed low ESR1 mRNA but variable PGR mRNA (Additional file 7: Figure S2), indicating the existence of a ER-/PgR+ phenotype.

\section{Characterized gene expression of ER-/PgR+/HER2- phenotype}

Having found that the ER-/PgR+ phenotype was shared between luminal-like and basal-like groups at the molecular level, we further sought the characterized genes for luminal-like and basal-like ER-/PgR+ subgroups. The original gene expression data were only available in cohort 4 , 
Table 2 Univariate and multivariate analysis of survival for ER and PgR subgroups

\begin{tabular}{|c|c|c|c|c|c|c|c|c|c|c|c|c|c|c|c|c|}
\hline \multirow[t]{3}{*}{ Subgroups } & \multicolumn{4}{|c|}{ Cohort 1: BCSS } & \multicolumn{4}{|c|}{ Cohort 2: RFS } & \multicolumn{4}{|c|}{ Cohort 3: RFS } & \multicolumn{4}{|c|}{ Cohort 4: DRFS } \\
\hline & \multicolumn{2}{|l|}{$\mathrm{HR}(95 \% \mathrm{Cl})$} & \multirow[t]{2}{*}{ P1 } & \multirow[t]{2}{*}{ P2 } & \multicolumn{2}{|l|}{$\overline{\mathrm{HR}}(95 \% \mathrm{Cl})$} & \multirow[t]{2}{*}{ P1 } & \multirow[t]{2}{*}{ P2 } & \multicolumn{2}{|c|}{$\mathrm{HR}(95 \% \mathrm{Cl})$} & \multirow[t]{2}{*}{ P1 } & \multirow[t]{2}{*}{ P2 } & \multicolumn{2}{|c|}{ HR (95 \% Cl) } & \multirow[t]{2}{*}{ P1 } & \multirow[t]{2}{*}{ P2 } \\
\hline & Univariate & Adjusted $^{a}$ & & & Univariate & Adjusted $^{a}$ & & & Univariate & Adjusted $^{\mathrm{b}}$ & & & Univariate & Adjusted $^{c}$ & & \\
\hline ER+/PgR+ & 1 (ref.) & 1 (ref.) & $<0.001^{d}$ & & 1 (ref.) & 1 (ref.) & $<0.001^{d}$ & & 1 (ref.) & 1 (ref.) & $0.006^{d}$ & & 1 (ref.) & 1 (ref.) & $<0.001^{d}$ & \\
\hline ER-/PgR+ & $\begin{array}{l}4.67(2.55- \\
8.57)\end{array}$ & $\begin{array}{l}3.26(1.71- \\
6.22)\end{array}$ & $<0.001$ & $<0.001$ & $\begin{array}{l}2.76(1.27- \\
5.99)\end{array}$ & $\begin{array}{l}2.61(1.20- \\
5.67)\end{array}$ & 0.017 & 0.016 & $\begin{array}{l}2.51(1.11- \\
5.68)\end{array}$ & $\begin{array}{l}2.68(1.10- \\
6.55)\end{array}$ & 0.028 & 0.030 & $\begin{array}{l}0.78(0.19- \\
3.27)\end{array}$ & $\begin{array}{l}1.09(0.26- \\
4.64)\end{array}$ & 0.79 & 0.90 \\
\hline ER-/PgR- & $\begin{array}{l}7.26(6.10- \\
8.64)\end{array}$ & $\begin{array}{l}4.12(3.33- \\
5.09)\end{array}$ & $<0.001$ & $<0.001$ & $\begin{array}{l}3.65(2.71- \\
4.92)\end{array}$ & $\begin{array}{l}4.10(3.03- \\
5.54)\end{array}$ & $<0.001$ & $<0.001$ & $\begin{array}{l}2.59(1.36- \\
4.93)\end{array}$ & $\begin{array}{l}2.51(1.21- \\
5.20)\end{array}$ & 0.003 & 0.013 & $\begin{array}{l}3.33(2.16- \\
5.15)\end{array}$ & $\begin{array}{l}3.66(2.15- \\
6.23)\end{array}$ & $<0.001$ & $<0.001$ \\
\hline
\end{tabular}

Here we provided both unadjusted and adjusted values of HR of BCSS/RFS/DRFS to show the survival effect of ER/PgR status

P1: Pairwise $P$ value for univariate analysis (by log-rank test). ER+/PgR+ group as reference

P2: Pairwise $P$ value for multivariate analysis (by Cox regression). ER+/PgR+ group as reference

$B C S S$ Breast cancer-specific survival, CI Confidence interval, DRFS Distant relapse-free survival, HR Hazard ratio, ref Reference, RFS Relapse-free survival

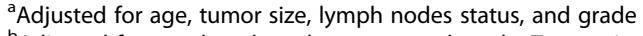

${ }^{\mathrm{b}}$ Adjusted for age, lymph nodes status, and grade. Tumor size is not adjusted because only half of cases have available information on it

'Adjusted for age, tumor size, lymph nodes status, grade, and pathological complete response

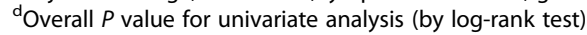


Table 3 Relationship between immunohistochemistry-based subgroups and PAM50-based intrinsic subtypes

\begin{tabular}{|c|c|c|c|c|c|c|c|c|c|c|c|c|}
\hline \multirow[b]{2}{*}{ IHC-based subgroup (HER2-) } & \multirow[b]{2}{*}{ Total (n) } & \multicolumn{11}{|c|}{ Gene-expression based subtype (by PAM50 classifier) } \\
\hline & & Luminal-A (n) & $\%$ & Luminal-B (n) & $\%$ & Basal (n) & $\%$ & HER2+ (n) & $\%$ & Normal-like (n) & $\%$ & $P$ \\
\hline Cohort 3 & & & & & & & & & & & & $<0.001$ \\
\hline $\mathrm{ER}+/ \mathrm{PgR}+$ & 391 & 179 & 45.8 & 168 & 43.0 & 20 & 5.1 & 7 & 1.8 & 17 & 4.3 & \\
\hline $\mathrm{ER}-/ \mathrm{PgR}+$ & 36 & 6 & 16.7 & 6 & 16.7 & 20 & 55.6 & 3 & 8.3 & 1 & 2.8 & \\
\hline $\mathrm{ER}-/ \mathrm{PgR}-$ & 280 & 9 & 3.2 & 27 & 9.6 & 207 & 73.9 & 25 & 8.9 & 12 & 4.3 & \\
\hline Cohort 4 & & & & & & & & & & & & $<0.001$ \\
\hline $\mathrm{ER}+/ \mathrm{PgR}+$ & 216 & 126 & 58.3 & 50 & 23.1 & 13 & 6.0 & 12 & 5.6 & 15 & 6.9 & \\
\hline $\mathrm{ER}-/ \mathrm{PgR}+$ & 17 & 3 & 17.6 & 1 & 5.9 & 11 & 64.7 & 1 & 5.9 & 1 & 5.9 & \\
\hline $\mathrm{ER}-/ \mathrm{PgR}-$ & 178 & 2 & 1.1 & 3 & 1.7 & 142 & 79.8 & 14 & 7.9 & 17 & 9.6 & \\
\hline
\end{tabular}

IHC Immunohistochemistry

but not in the remaining three cohorts. Figure 2 shows the differential expression of candidate genes across different intrinsic subtypes within the ER-/PgR+ phenotype. Higher expression of TFF1 and GREB1 is significantly associated with luminal-like (Mann-Whitney test $P=0.005$ and $P=$ 0.02 , respectively, Fig. 2a), while increased expression of CK5 (KRT5) or EGFR tended to be associated with basal-like (Mann-Whitney test $P=0.05$ and $P=0.007$, respectively, Fig. 2b). The combination of TFF1 with CK5 or EGFR significantly discriminated luminal-like $\mathrm{ER}-/ \mathrm{PgR}+$ from basal-like ER-/PgR+ (Fig. 2c). Of note, the basal-like subtype within ER-/PgR+ did not show claudin-low or CDH1low features compared with the luminal-like subtype.

Refine the subtypes within the ER-/PgR+/HER2- phenotype by IHC markers

Based on the findings of characterized gene expression for luminal-like and basal-like ER-/PgR+, we further

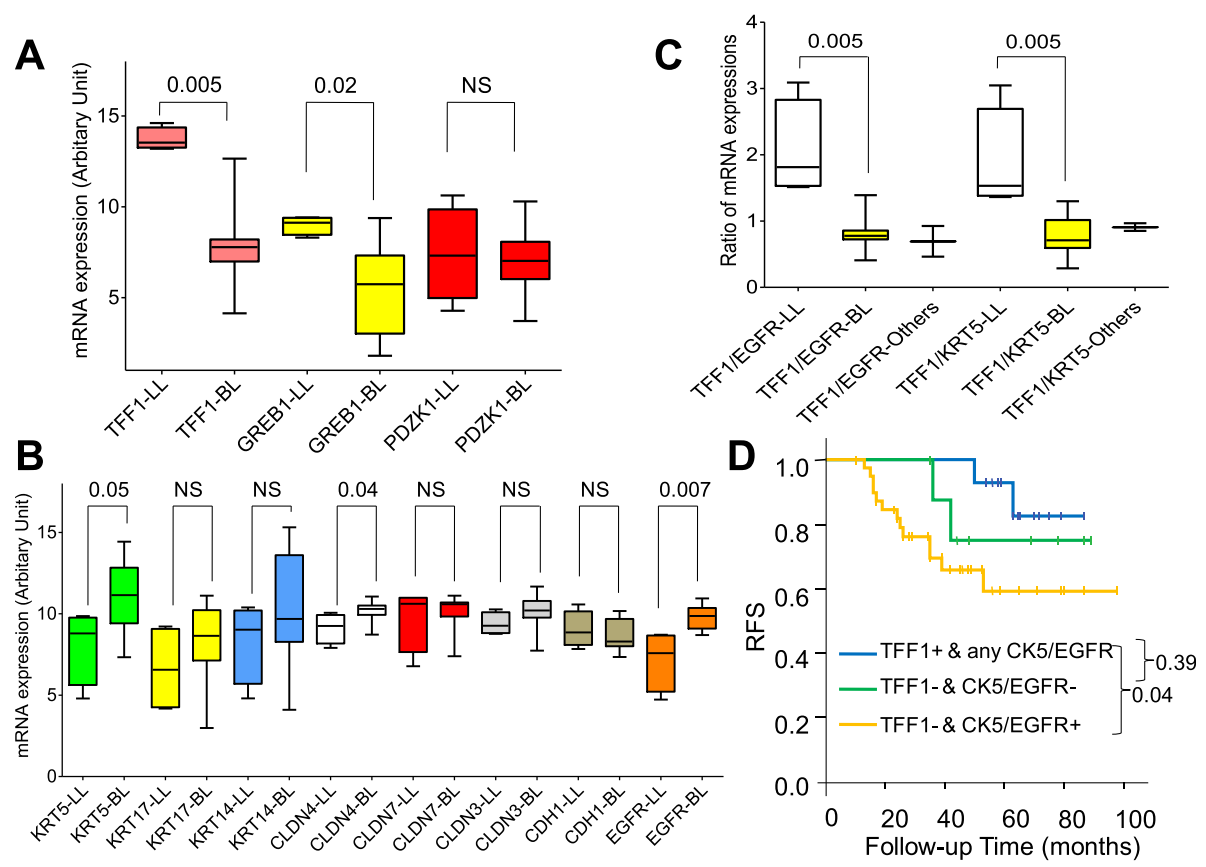

Fig. 2 Expression of featured genes in tumors with the ER-/PgR+ phenotype. Box plots of expression of estrogen-responsive genes (a) and basal, claudins, and mesenchymal stem genes (b) for subtypes within ER-/PgR+/HER2- cases from cohort 4. (c) Ratio of TFF1 to EGFR or to CK5 for basal-like $(n=4)$, luminal-like $(n=11)$, and other subtypes $(n=2)$. $P$ values are for comparisons between luminal-like and basal-like by Mann-Whitney test. The probe sets used for gene expression are 205009_at for TFF1, 205862_at for GREB1, 205380_at for PDZK1, 201820_at for KRT5, 205157_S_at for KRT17, 209351_at for KRT14, 201428_at for CLDN4, 202790_at for CLDN7, 203953_s_at for CLDN3, 201130_s_at for CDH1, and 201983_s_at for EGFR. (d) Kaplan-Meier estimates of relapse-free survival according to subgroups within the ER-/PgR+ phenotype using immunohistochemistry-based TFF1 (pS2), KRT5 (CK5), and EGFR. Three groups were defined as follows: luminal-like is defined as TFF1-positive and any CK5 and/or EGFR staining; basal-like is defined as TFF1-negative and positive for at least one marker of CK5 and EGFR; the remaining cases are in the undetermined group. Log-rank $P$ values for pairwise comparison are shown. BL, Basal-like; LL, Luminal-like; NS, Not significant 
validated three characterized markers by IHC to determine an individual case as a certain subtype by a feasible IHC method. Because EGFR and CK5 (coded by KRT5) expression rates are not high (55-65\%) in basal-like cases [25, 42], we employed both EGFR and CK5 to single out basal-like to a large extent. We performed this analysis in the 64 ER-/PgR+/HER2- cases from our single institute between 2005 and 2011 because we could obtain their tissue samples for IHC assay but could not get formalinfixed paraffin-embedded samples in cohorts 1,3 , and 4 . We characterized the 64 cases into three groups by expression of TFF1, EGFR, and CK5. Basal-like and luminallike subtypes were identified and constituted $63 \%$ (40 of 64; $95 \%$ CI, 50-74 \%) and $23 \%$ (15 of 64; $95 \%$ CI, 14$36 \%$ ) of the tumors studied, respectively (Additional file 2 : Table S1). The basal-like subgroup displayed the worst prognosis relative to the other two subgroups while the luminal-like cases tended to have the most favorable RFS
(Fig. 2d). After adjustment for other prognostic factors such as age at diagnosis, tumor size, node status, and grade, the three-marker defined subgroup was an independent prognostic factor for relapse (HR of $2.4 ; 95 \% \mathrm{CI}$, 1.17-5.03; $P=0.017$ ).

\section{Sensitivity to endocrine therapy of subtypes within ER-/PgR+/HER2- phenotype}

Survival analysis in 55 out of the $64 \mathrm{ER}-/ \mathrm{PgR}+$ cases from FDUSCC according to adjuvant endocrine therapy is shown in Additional file 8: Table S5. Patients with a luminal-like ER-/PgR+ subtype benefited more from sufficient adjuvant endocrine therapy (defined as undergoing cumulative endocrine treatment for more than one year) than insufficient treatment (less than one year or no endocrine therapy; $\log$-rank $P=0.06$. Fig. 3a). In contrast, the basal-like subgroup did not benefit from endocrine therapy (log-rank $P=0.61$. Fig. $3 \mathrm{~b})$. Because
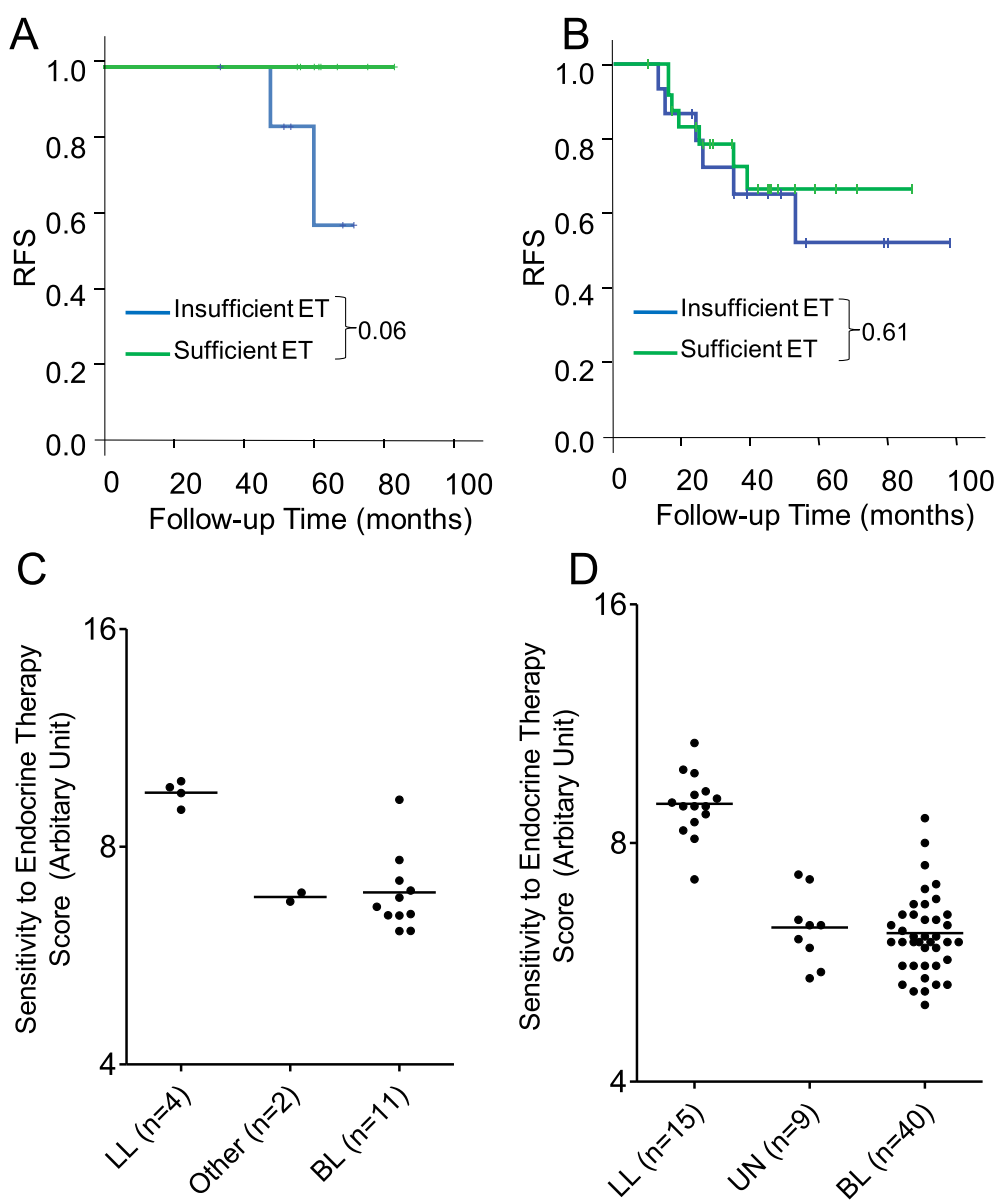

Fig. 3 Sensitivity to endocrine therapy of subtypes within ER-/PgR+ phenotype. Kaplan-Meier estimates of RFS according to sufficient endocrine therapy or not in the luminal-like (a) and basal-like (b) subgroup in 55 out of the 64 ER-/PgR+/HER2- cases. An endocrine therapy sensitivity score was also calculated according to the subgroups within the ER-/PgR+ group in the 17 cases from cohort 4 (c) and in the 64 cases from cohort 2 (d). The subgroups within the ER-/PgR+ were evaluated by PAM50 in cohort 4 and by immunohistochemistry in the 64 cases. $P$ values of sensitivity score between luminal-like and basal-like are $<0.0001$ for both sets (Mann-Whitney test). LL, Luminal-like; UN, Undetermined; BL, Basal-like 
of limited cases and rare events, the survival outcome of multivariate analysis was unavailable.

To find more evidence to support the above findings, we evaluated the sensitivity to endocrine therapy at the molecular level by calculating the ER group score (reflects the endocrine responsiveness) in two sets of ER-/PgR+/HER2cases. The first set was from cohort 4, including 17 cases, and the second set was from cohort 2, including 64 cases. It seemed that luminal-like cases (identified by either PAM50 classifier or IHC-based TFF1/CK5/EGFR makers) had a higher score of sensitivity to endocrine therapy compared with basal-like cases (Mann-Whitney test $P$ values $<0.0001$ for both sets; Fig. 3c, d). When we combined the two sets using a meta-analytic approach (metaanalysis of continuous outcomes, Hedges' g method), the luminal-like cases had higher score of sensitivity to endocrine therapy compared with basal-like cases (standardized mean difference of 3.45 with $95 \% \mathrm{CI}, 2.65-4.26 ; P<0.0001$, by a fixed-effects method). Further analysis showed that basal-like ER-/PgR+/HER2- cases had endocrine therapy sensitivity scores as low as those in triple-negative cases $(P=0.80$, by a fixed-effects method).

\section{Discussion}

In the present study, we systemically investigated the clinicopathologic features and molecular essence of a clinically rare but biologically occurring ER-/PgR+/HER2phenotype. We revealed that the clinicopathologic features and survival outcomes of this phenotype fell in between $\mathrm{ER}+/ \mathrm{PgR}+$ and $\mathrm{ER}-/ \mathrm{PgR}-$ and were more similar to the ER-/PgR- phenotype. For the intrinsic subtype of ER-/ PgR+ tumors, about $30 \%$ were luminal-like and $60 \%$ were basal-like. Moreover, we developed a feasible IHCbased method using three markers, TFF1, CK5, and EGFR, to determine the prognosis-relevant subtype of each ER-/ $\mathrm{PgR}+$ case, which may assist oncologists in making treatment decisions. ER-/PgR+ cases with basal-like characteristics may eliminate long-term but ineffective endocrine therapy and lead to individualized chemotherapy.

In our series, the majority of ER-/PgR+ tumors occurred in younger women with poorly differentiated tumors, which have been observed in triple-negative cases [43]. At the molecular level, about $60 \%$ were associated with a basal-like subtype, while only less than $30 \%$ showed luminal features. Currently, routine clinical evaluation of subtype is most valuable in predicting the response to targeted therapy. Clinical guidelines, such as the St. Gallen consensus, recommend tailoring adjuvant systemic treatment according to subtypes [11]. However, the ER-/PgR+ phenotype is not mentioned in the 2013 St. Gallen consensus, and the recommended treatment is therefore undetermined. Although the ER-/PgR+ phenotype belongs to the "hormone receptor-positive" group and is suggested to use endocrine therapy, its response to endocrine therapy is low $[3,4,12]$.
According to a collaborative meta-analysis of individual patient data from 20 trials $(n=21,457)$ in early breast cancer of about 5 years of tamoxifen versus no adjuvant tamoxifen by Early Breast Cancer Trialists' Collaborative Group [4], the rate ratios were 0.63 (standard error, 0.03) for $\mathrm{ER}+/ \mathrm{PgR}+$ disease $(P<0.00001)$ but 0.90 (standard error, 0.10$)$ for $\mathrm{ER}-/ \mathrm{PgR}+$ disease $(P=0.35)$. We herein demonstrated that the majority of $\mathrm{ER}-/ \mathrm{PgR}+$ cases were actually basal-like, therefore indicating that treatment of $\mathrm{ER}-/ \mathrm{PgR}+$ cases with long-term endocrine therapy for 5 years or even more is questionable.

Being able to identify the luminal-like subgroup within the ER-/PgR+ phenotype is important. Our study provides, for the first time, an effective and feasible IHC method to distinguish the intrinsic subtype within the ER-/PgR+ phenotype using three markers, TFF1, CK5, and EGFR. TFF1 is an indicator of the functional estrogen-responsive pathway and improves the response to tamoxifen [44]. KRT5 and EGFR are identified as reliable basal markers [25]. Moreover, we identified a significant difference in the sensitivity to endocrine therapy between luminal-like ER-/PgR+ and basal-like ER-/PgR+. Basal-like $\mathrm{ER}-/ \mathrm{PgR}+$ cases obtained limited benefit from endocrine therapy, while luminal-like ER-/PgR+ cases probably benefited from endocrine therapy despite of ER loss. There are some potential explanations for this. First, in these cases, ER-negativity is falsely negative [5]. Technical failure in ER detection made it difficult to detect positive ER even after re-assessment by IHC. Second, strong evidence exists for the presence of plasma membrane ER (only nuclear staining of ER is recognized as ER-positivity according to the ASCO/CAP guideline [12]). When estrogen binds cell surface ER, membrane-initiated stimulation is able to induce and potentiate the genomic activation of $\mathrm{PgR}$ expression $[45,46]$. In this situation, endocrine therapy by antagonizing or reducing estrogen may also work.

Our study has some limitations. First, we excluded HER2+ cases and thus our findings could not be applicable in the ER-/PgR+/HER2+ phenotype. Second, although it is better to use the same survival endpoint (BCSS, RFS, or DRFS) for analysis, unfortunately, the various cohorts provide different endpoints and it is impossible to use the same endpoint for analysis. Third, because of limited ER-/PgR+/HER2- cases included in analysis of sensitivity to endocrine therapy and rare survival events, it is still too early to conclude the causal association between basal-like $\mathrm{ER}-/ \mathrm{PgR}+$ tumors and limited benefit from endocrine therapy. Finally, our study is biased by its retrospective nature. However, due to the very low incidence of the ER-/PgR+/HER2- phenotype, it is impractical to conduct a large-scale prospective trial to test our hypothesis; we therefore must rely on data from the present large retrospective study. Our study uses the data from some prospective observational cohorts and provides a piece 
of state-of-the-art evidence describing the molecular essence of ER-/PgR+ and how to recognize the subtype of a $\mathrm{ER}-/ \mathrm{PgR}+$ case using an IHC assay.

\section{Conclusion}

In conclusion, the majority of the ER-/PgR+/HER2phenotype breast cancer cases are basal-like and a minority is luminal-like. Detecting immunohistochemical TFF1, CK5, and EGFR may help to identify the intrinsic subgroups within this phenotype. Basal-like ER-/PgR+ tumors may obtain limited benefit from endocrine therapy and further large-scale studies will be necessary to validate our findings.

\section{Additional files}

Additional file 1: Figure S1. The study flowchart diagram. FDUSCC, Fudan University Shanghai Cancer Center; NCT, Neoadjuvant chemotherapy; SEER, Surveillance, Epidemiology and End Results program. (PPTX 111 kb)

Additional file 2: Table S1. Characteristics of the 64 ER-/PgR+/HER2phenotype cases from FDUSCC. (DOC $49 \mathrm{~kb}$ )

Additional file 3: Table S2. Genes and probe sets used to characterize subgroups within ER-/PgR+/HER2- phenotype. (DOC $40 \mathrm{~kb}$ )

Additional file 4. Supplemental Methods. (DOC $35 \mathrm{~kb}$ )

Additional file 5: Table S3. PCR Primers of ER group genes and reference genes. (DOC $32 \mathrm{~kb}$ )

Additional file 6: Table S4. Comparison of characteristics among ER +/PgR+, ER-/PgR+, and ER-/PgR- phenotypes. (DOC 58 kb)

Additional file 7: Figure S2. ER and PgR expression in the ER-/PgR +/HER2- phenotype in cohort 4. PP, ER+ and PgR+; NP, ER- and PgRt; NN, ER- and PgR-. (PPTX $79 \mathrm{~kb}$ )

Additional file 8: Table S5. Survival benefit from adjuvant endocrine therapy in 55 out of the 64 ER-/PgR+/HER2- cases. (DOC $30 \mathrm{~kb}$ )

\section{Abbreviations}

ASCO/CAP: American Society of Clinical Oncology/College of American Pathologists; BCSS: Breast cancer-specific survival; Cls: Confidence intervals; CKs: Cytokeratins; DRFS: Distant relapse-free survival; ER: Estrogen receptor; FDUSCC: Fudan University Shanghai Cancer Center; HRs: Hazard ratios; IHC: Immunohistochemistry; PgR: Progesterone receptor; RFS: Relapse-free survival; SEER: Surveillance, Epidemiology, and End Results.

\section{Competing interest}

The authors have declared that no competing interests exist.

\section{Authors' contributions}

Conceived and designed: KDY and ZMS. Performed the research: KDY and YZJ. Analyzed the data: KDY, YZJ, SH. Contributed reagents/materials/analysis tools: KDY, YZJ, SH, and ZMS. Wrote the paper: KDY, YZJ, SH, and ZMS. All authors read and approved the final manuscript.

\section{Acknowledgements}

This work was supported by National Natural Science Foundation of China (81001169, 81370075), Training Plan of Excellent Talents in Shanghai Municipality Health System (XYQ2013101), Research and Innovation Project of Shanghai Municipal Education Commission, Shanghai International Science and Technique Cooperation Foundation (12410707700), International S\&T Cooperation Program of China (ISTCP No. 09), and the Shanghai Key Laboratory of Breast Cancer (12DZ2260100). The funders had no role in the study design, data collection and analysis, decision to publish, or preparation of the manuscript.
Received: 28 July 2015 Accepted: 18 September 2015

Published online: 05 October 2015

\section{References}

1. Cui X, Schiff R, Arpino G, Osborne CK, Lee AV. Biology of progesterone receptor loss in breast cancer and its implications for endocrine therapy. J Clin Oncol. 2005;23:7721-35.

2. Rakha EA, El-Sayed ME, Green AR, Paish EC, Powe DG, Gee J, et al. Biologic and clinical characteristics of breast cancer with single hormone receptor positive phenotype. J Clin Oncol. 2007;25:4772-8.

3. Yu KD, Di GH, Wu J, Lu JS, Shen KW, Liu GY, et al. Breast cancer patients with estrogen receptor-negative/progesterone receptor-positive tumors: being younger and getting less benefit from adjuvant tamoxifen treatment. J Cancer Res Clin Oncol. 2008;134:1347-54.

4. Davies C, Godwin J, Gray R, Clarke M, Cutter D, Darby S, et al. Relevance of breast cancer hormone receptors and other factors to the efficacy of adjuvant tamoxifen: patient-level meta-analysis of randomised trials. Lancet. 2011;378:771-84

5. De Maeyer L, Van Limbergen E, De Nys K, Moerman P, Pochet N, Hendrickx $W$, et al. Does estrogen receptor negative/progesterone receptor positive breast carcinoma exist? J Clin Oncol. 2008;26:335-6. Author reply 336-8.

6. Nadji M, Gomez-Fernandez C, Ganjei-Azar P, Morales AR. Immunohistochemistry of estrogen and progesterone receptors reconsidered: experience with 5,993 breast cancers. Am J Clin Pathol. 2005;123:21-7.

7. Maleki Z, Shariat S, Mokri M, Atri M. ER-negative/PR-positive breast carcinomas or technical artifacts in immunohistochemistry? Arch Iran Med. 2012;15:366-9

8. Rhodes A, Jasani B. The oestrogen receptor-negative/progesterone receptor-positive breast tumour: a biological entity or a technical artefact? J Clin Pathol. 2009:62:95-6.

9. Ng CH, Pathy NB, Taib NA, Mun KS, Rhodes A, Yip CH. The estrogen receptor negative-progesterone receptor positive breast carcinoma is a biological entity and not a technical artifact. Asian Pac J Cancer Prev. 2012;13:1111-3.

10. Borras M, Lacroix M, Legros N, Leclerca G. Estrogen receptor-negative/ progesterone receptor-positive Evsa-T mammary tumor cells: a model for assessing the biological property of this peculiar phenotype of breast cancers. Cancer Lett. 1997;120:23-30.

11. Goldhirsch A, Winer EP, Coates AS, Gelber RD, Piccart-Gebhart M, Thurlimann $B$, et al. Personalizing the treatment of women with early breast cancer: highlights of the St Gallen International Expert Consensus on the Primary Therapy of Early Breast Cancer 2013. Ann Oncol. 2013;24:2206-23.

12. Hammond ME, Hayes DF, Dowsett M, Allred DC, Hagerty KL, Badve S, et al. American Society of Clinical Oncology/College Of American Pathologists guideline recommendations for immunohistochemical testing of estrogen and progesterone receptors in breast cancer. J Clin Oncol. 2010;28:2784-95.

13. Itoh M, Iwamoto T, Matsuoka J, Nogami T, Motoki T, Shien T, et al. Estrogen receptor (ER) mRNA expression and molecular subtype distribution in ER-negative/progesterone receptor-positive breast cancers. Breast Cancer Res Treat. 2014;143:403-9.

14. Sorlie T, Tibshirani R, Parker J, Hastie T, Marron JS, Nobel A, et al. Repeated observation of breast tumor subtypes in independent gene expression data sets. Proc Natl Acad Sci U S A. 2003;100:8418-23.

15. Haibe-Kains B, Desmedt C, Loi S, Culhane AC, Bontempi G, Quackenbush J, et al. A three-gene model to robustly identify breast cancer molecular subtypes. J Natl Cancer Inst. 2012;104:311-25.

16. Hatzis C, Pusztai L, Valero V, Booser DJ, Esserman L, Lluch A, et al. A genomic predictor of response and survival following taxane-anthracycline chemotherapy for invasive breast cancer. JAMA. 2011;305:1873-81.

17. National Cancer Institute. Surveillance, Epidemiology, and End Results Program. Turning Cancer Data Into Discovery. http://seer.cancer.gov/seerstat/. Accessed date: September 30, 2014

18. Yu KD, Di GH, Wu J, Lu JS, Shen KW, Shen ZZ, et al. Development and trends of surgical modalities for breast cancer in China: a review of 16-year data. Ann Surg Oncol. 2007;14:2502-9.

19. Goldhirsch A, Wood WC, Gelber RD, Coates AS, Thurlimann B, Senn HJ Progress and promise: highlights of the international expert consensus on the primary therapy of early breast cancer 2007. Ann Oncol. 2007;18:1133-44.

20. Wolff AC, Hammond ME, Schwartz JN, Hagerty KL, Allred DC, Cote RJ, et al. American Society of Clinical Oncology/College of American Pathologists 
guideline recommendations for human epidermal growth factor receptor 2 testing in breast cancer. J Clin Oncol. 2007;25:118-45.

21. Shi L, Reid LH, Jones WD, Shippy R, Warrington JA, Baker SC, et al. The MicroArray Quality Control (MAQC) project shows inter- and intraplatform reproducibility of gene expression measurements. Nat Biotechnol. 2006;24:1151-61.

22. Parker JS, Mullins M, Cheang MC, Leung S, Voduc D, Vickery T, et al. Supervised risk predictor of breast cancer based on intrinsic subtypes. J Clin Oncol. 2009;27:1160-7.

23. Symmans WF, Hatzis C, Sotiriou C, Andre F, Peintinger F, Regitnig P, et al. Genomic index of sensitivity to endocrine therapy for breast cancer. J Clin Oncol. 2010;28:4111-9.

24. Dunbier AK, Anderson H, Ghazoui Z, Folkerd EJ, A'Hern R, Crowder RJ, et al. Relationship between plasma estradiol levels and estrogen-responsive gene expression in estrogen receptor-positive breast cancer in postmenopausal women. J Clin Oncol. 2010;28:1161-7.

25. Nielsen TO, Hsu FD, Jensen K, Cheang M, Karaca G, Hu Z, et al. Immunohistochemical and clinical characterization of the basal-like subtype of invasive breast carcinoma. Clin Cancer Res. 2004;10:5367-74.

26. Alshareeda AT, Soria D, Garibaldi JM, Rakha E, Nolan C, Ellis IO, et al. Characteristics of basal cytokeratin expression in breast cancer. Breast Cancer Res Treat. 2013;139:23-37.

27. Lehmann BD, Bauer JA, Chen X, Sanders ME, Chakravarthy AB, Shyr Y, et al. Identification of human triple-negative breast cancer subtypes and preclinical models for selection of targeted therapies. J Clin Invest. 2011;121:2750-67.

28. Hennessy BT, Gonzalez-Angulo AM, Stemke-Hale K, Gilcrease MZ, Krishnamurthy S, Lee JS, et al. Characterization of a naturally occurring breast cancer subset enriched in epithelial-to-mesenchymal transition and stem cell characteristics. Cancer Res. 2009;69:4116-24.

29. Paik S, Shak S, Tang G, Kim C, Baker J, Cronin M, et al. A multigene assay to predict recurrence of tamoxifen-treated, node-negative breast cancer. N Engl J Med. 2004;351:2817-26.

30. Esteva FJ, Sahin AA, Cristofanilli M, Coombes K, Lee SJ, Baker J, et al. Prognostic role of a multigene reverse transcriptase-PCR assay in patients with node-negative breast cancer not receiving adjuvant systemic therapy. Clin Cancer Res. 2005;11:3315-9.

31. Akashi-Tanaka S, Shimizu C, Ando M, Shibata T, Katsumata N, Kouno T, et al. 21-Gene expression profile assay on core needle biopsies predicts responses to neoadjuvant endocrine therapy in breast cancer patients. Breast. 2009;18:171-4.

32. Detre S, King N, Salter J, MacLennan K, McKinna JA, Dowsett M. Immunohistochemical and biochemical analysis of the oestrogen regulated protein pS2, and its relation with oestrogen receptor and progesterone receptor in breast cancer. J Clin Pathol. 1994;47:240-4.

33. Chen $\mathrm{S}$, Chen $\mathrm{CM}$, Yu KD, Yang WT, Shao ZM. A prognostic model to predict outcome of patients failing to achieve pathological complete response after anthracycline-containing neoadjuvant chemotherapy for breast cancer. J Surg Oncol. 2012;105:577-85.

34. Abo-Zaid G, Guo B, Deeks JJ, Debray TP, Steyerberg EW, Moons KG, et al. Individual participant data meta-analyses should not ignore clustering. J Clin Epidemiol. 2013;66:865-73. e864.

35. Higgins JP, Thompson SG, Deeks JJ, Altman DG. Measuring inconsistency in meta-analyses. BMJ. 2003;327:557-60.

36. Newcombe RG. Two-sided confidence intervals for the single proportion: comparison of seven methods. Stat Med. 1998;17:857-72.

37. White IR. Multivariate random-effects meta-analysis. Stata J. 2009:9:40-56.

38. Jackson D, White IR, Thompson SG. Extending DerSimonian and Laird's methodology to perform multivariate random effects meta-analyses. Stat Med. 2010;29:1282-97.

39. Colditz GA, Rosner BA, Chen WY, Holmes MD, Hankinson SE. Risk factors for breast cancer according to estrogen and progesterone receptor status. J Natl Cancer Inst. 2004;96:218-28.

40. Perou CM, Sorlie T, Eisen MB, van de Rijn M, Jeffrey SS, Rees CA, et al. Molecular portraits of human breast tumours. Nature. 2000;406:747-52.

41. Hefti MM, Hu R, Knoblauch NW, Collins LC, Haibe-Kains B, Tamimi RM, et al. Estrogen receptor negative/progesterone receptor positive breast cancer is not a reproducible subtype. Breast Cancer Res. 2013;15:R68.

42. Banerjee S, Reis-Filho JS, Ashley S, Steele D, Ashworth A, Lakhani SR, et al. Basal-like breast carcinomas: clinical outcome and response to chemotherapy. J Clin Pathol. 2006;59:729-35.
43. Metzger-Filho O, Tutt A, de Azambuja E, Saini KS, Viale G, Loi S, et al. Dissecting the heterogeneity of triple-negative breast cancer. J Clin Oncol. 2012;30:1879-87.

44. Soubeyran I, Quenel N, Coindre JM, Bonichon F, Durand M, Wafflart J, et al. pS2 protein: a marker improving prediction of response to neoadjuvant tamoxifen in post-menopausal breast cancer patients. Br J Cancer. 1996;74:1120-5.

45. Levin ER. Plasma membrane estrogen receptors. Trends Endocrinol Metab. 2009;20:477-82.

46. Sa SI, Pereira PA, Malikov V, Ferreira IM, Madeira MD. Role of plasma membrane estrogen receptors in mediating the estrogen induction of progesterone receptors in hypothalamic ventromedial neurons. J Comp Neurol. 2014;522:298-307.

\section{Submit your next manuscript to BioMed Central and take full advantage of:}

- Convenient online submission

- Thorough peer review

- No space constraints or color figure charges

- Immediate publication on acceptance

- Inclusion in PubMed, CAS, Scopus and Google Scholar

- Research which is freely available for redistribution 